\title{
Sobre la flexibilidad laboral en Colombia y la precarización del empleo*
}

\section{Job flexibility in Colombia and job insecurity}

\author{
María Alejandra Gómez Vélez** \\ Universidad Pontificia Bolivariana, \\ Colombia
}

Recibido: 10 de diciembre de 2012 Revisado: 12 de Marzo de 2013 Aceptado: 15 de abril de 2013

\section{Resumen}

El presente artículo hace parte del proceso de revisión documental de la investigación doctoral: Sentidos y significados del trabajo en el escenario laboral flexible. Una aproximación al contexto de MedellínColombia. Objetivo: indagar sobre la flexibilidad laboral en Colombia y su contribución a la precarización del empleo. Método: revisión de literatura. Procedimiento: se realizó una revisión de artículos relacionados, se consultaron investigaciones realizadas en Colombia, medios de comunicación masivos y fuentes gubernamentales de información. Se encontró en los resultados que la flexibilidad laboral en Colombia, se ha impuesto como una exigencia de las organizaciones a los Estados en su búsqueda de competitividad y sobrevivencia, pero que ha traído pérdidas significativas a la protección legal que tenían los trabajadores, incrementando cada vez más la precarización del empleo. En Colombia la flexibilidad laboral se presenta a través de los contratos comerciales con empresas temporales, cooperativas de trabajo asociado y contratos de prestación de servicios; además de la utilización de contratos laborales a término fijo. La conclusión, a modo general, es que el incremento de la utilización de estas modalidades de relación laboral se aumenta año a año y se incrementa la pérdida de

* Artículo de investigación.

** Correspondencia: María Alejandra Gómez Vélez. Psicóloga, Especialista en Psicología Organizacional, Magister en Ciencias Sociales y Doctoranda en Psicología de la Universidad Católica de Argentina. Miembro de la Red Iberoamericana de Investigadores en Factores de Riesgo Psicosocial Laboral-RIPSOL. Este artículo hace parte de la revisión de contexto necesaria para la tesis doctoral. Dirección postal: Facultad de Psicología Universidad Pontificia Bolivariana, Circular 1 No. 70-01, Bloque 7. Medellín, Colombia. Correo electrónico: alejandra.gomez@upb.edu.co 
calidad del trabajo y su estabilidad, con su respectiva disminución de calidad de vida y bienestar de los trabajadores y de sus familias, es así que, la consecución del empleo se constituye en una forma de responder a la necesidad de sobrevivencia. Es de resaltar que los empleadores contratan y desvinculan al personal bajo un criterio básicamente económico, incentivando en esta lógica de relación contractual el exceso de poder del más fuerte, es decir del empleador.

Palabras clave: flexibilidad laboral, precariedad del empleo, contrato laboral, competitividad.

\section{Abstract}

This document is part of the doctoral research: Sense and Meaning of Job in a Flexible Job Market Environment. Approach to the Medellin - Colombia case. Objective: To study the flexible job market in Colombia and its contribution to job quality deterioration. Methodology: Literature review. Procedure: review of peer articles and research done in Colombia, media releases and government available data and information. The results show that job market flexibility in Colombia has emerged as a demand from businesses to government in their pursuing for competitiveness and survival. However, it has brought in with it a significant loss of legal protection for workers, increasing the deterioration of job quality. The job market flexibility in Colombia is implemented through contracts with agency employers, workers' cooperatives and defined body work contracts, on top of definite term employment. In conclusion, and generally speaking, the use of this type of employment contract is rising every year and increases the loss of job quality and lack of stability, with the consequent deterioration on quality of life and well-being of workers and their families. In this environment, getting a job becomes a matter of survival. It is worth to note that employers hire and fire personnel under basic economic criteria, encouraging a job market ruled by the excess of power of the strongest, the employer.

Keywords: Job flexibility, job insecurity, employment contract, competitiveness.

\section{Flexibilidad laboral en Colombia y precarización del empleo}

Este artículo hace parte de la revisión de contexto que se viene realizando para la tesis doctoral: Sentidos y significados del trabajo en el escenario laboral flexible. Una aproximación al contexto de Medellín-Colombia. Esta investigación surge del interés académico y social de profundizar en una reciente realidad producida a partir de la globalización de la economía, la de la flexibilización laboral, que ha dado un giro a las relaciones laborales y por lo tanto también a las sociales.

Los cambios producidos en el mundo, que se consolidan a partir de la década de los años ochenta, han traído unas formas nuevas de relacionarse, de valorar el vínculo laboral, de lidiar con la incertidumbre reinante, de identificarse temporariamente a una organización, de considerar la estabilidad laboral como un tema del pasado y de conceptuar que el trabajo es un medio esencialmente para la sobrevivencia, particularmente para aquellos que tienen menos nivel de empleabilidad.

La globalización ha traído la flexibilidad laboral y con ella la pérdida de estabilidad laboral, y para muchos, la calidad del empleo. Este cambio en las relaciones contractuales ha traído unas formas de interacción que inciden en la vida diaria de las personas, en su concepción del trabajo y en su búsqueda agobiante de construir un proyecto de vida respaldado en los ingresos que permite el trabajo, cuando lo permite. 
Básicamente en Colombia se cuenta con empresas temporales, cooperativas de trabajo asociado, contratos por prestación de servicios y contratos fijos, que buscan el fortalecimiento de las organizaciones. El aumento de estas modalidades de contratación laboral y comercial se incrementa cada año, y se pactan tanto para personal no calificado como para el calificado. Estudiar e investigar este tema es hoy una necesidad sentida por los efectos que sobre las condiciones de vida tienen estas formas de vinculación al trabajo y por las repercusiones en el mediano y largo plazo para las mismas organizaciones, con relación a su propio futuro en materia de las personas que se comprometen o no a constituirlas.

\section{De la flexibilidad laboral a la precarización}

La flexibilidad laboral se ha propuesto como una salida a las preocupaciones de las organizaciones por su competitividad y viabilidad económica, a partir de la globalización de la economía. Desde 1980 la flexibilidad laboral es una forma de relacionarse cada vez más entre organizaciones y trabajadores, y los efectos recién se empiezan a estudiar en Colombia.

Como parte de sus medidas para recortar los gastos y de sus esfuerzos para mejorar su competitividad, las empresas trabajan cada vez más con un pequeño núcleo de trabajadores asalariados en condiciones regulares de empleo y una periferia cada vez mayor de trabajadores «no normalizados» o «atípicos» en diferentes tipos de lugares de trabajo dispersados en distintas ubicaciones, y algunas veces en distintos países. Estas medidas suelen incluir acuerdos de contratación externa o subcontratación y relaciones laborales más flexibles e informales (OIT. Conferencia Internacional del Trabajo 90.a reunión., 2002. p. 7.)

Con la globalización surge el concepto de la flexibilización laboral, identificándose con mecanismos jurídicos, reformas y estrategias, y su objetivo se ha centrado en quitar rigidez a la legislación laboral, con el fin de permitir que el trabajo se acomode y se adapte fácilmente a las necesidades y conveniencias del sistema productivo. Desde aquí se busca alcanzar mayores oportunidades de competitividad entre las empresas, lo paradójico es que esto se ha convertido en una frustración para los trabajadores que anhelan tener un empleo, estabilidad laboral y obtener mejores beneficios laborales y sociales, es decir, que aspiran a un trabajo decente.

Para la OIT y sus mandantes, el trabajo decente no es una norma sino una meta que ha de lograrse progresivamente. Aplicar un método progresivo implicaría comenzar en el extremo informal del continuo económico, en el que se ha creado la mayoría de los nuevos empleos en los últimos años, y promover la transición dentro de ese continuo hacia el extremo formal de la economía y del trabajo decente y protegido. Ello sería también parte integrante de un enfoque de la reducción de la pobreza basado en el trabajo decente (OIT. Conferencia Internacional del Trabajo 90.a reunión 2002. p. 10.)

Señala Fernández (2006, p. 5), que el surgimiento de las políticas flexibilizadoras del mercado de trabajo se enmarca dentro de las reformas de carácter neoliberal que se implementaron desde fines de los años setenta en los países desarrollados con el objetivo de responder a los principales problemas que aquejaban a las sociedades de entonces, como eran el desempleo, el déficit fiscal y la estanflación. Agrega el autor que estas políticas han llevado a transformaciones importantes en el mundo laboral, y que se pueden observar en el deterioro de las condiciones de vida de los trabajadores, en la pérdida de poder adquisitivo y de la menor participación en el ingreso nacional, además de una creciente percepción de inseguridad laboral que se sostiene en la precarización de las condiciones de trabajo. Señala Fernández (2006), que todo esto se observa en la generalización de contratación a tiempo parcial y en la disminución de los empleos seguros y estables. Asegura el autor que fue a finales de los años ochenta, que muchos gobiernos latinoamericanos emplearon políticas que se caracterizaban por lo que se conoce como "Consenso de Washington". 
Y enfatiza que uno de los temas que se plantean en este consenso es el de la desregulación de todos los mercados que incluía al mercado laboral. El principal propósito dice, era el de eliminar aquellas rigideces que no permitían una asignación eficiente de los recursos, en este caso el trabajo.

Paz y Piedrahita (2005) afirman que es posible diferenciar dos fuerzas cuando de flexibilidad laboral se trata, una que predomina y que atribuye efectos positivos a toda propuesta que tiene por propósito la adaptación empresarial que consiste en la supresión de normas sobre el trabajo asalariado, de las cuales se dice que son rígidas y le quitan el movimiento que deben tener las empresas para ganar y poder sobrevivir. Esto muestra, dicen los autores, que la existencia de normas supone un obstáculo para la utilización de las empresas del recurso humano, a su vez, que le atribuyen costos sociales que no permiten la contratación laboral, es decir, como si la protección legal perjudicara a los trabajadores. Esta es la opción de la ideología de la desregulación, afirman, que interpreta a la desregulación, como la forma de lograr el bienestar social. La otra fuerza, señalan, invita a reflexionar sobre las nuevas formas de crear normas para el trabajo asalariado, es decir, de lo que se trata es de plantear normas que sean versátiles con las necesidades de éxito y sobrevivencia de las empresas, pero no por ello que supriman los derechos de los trabajadores.

Ante las presiones del mundo globalizado, los Estados han realizado una serie de modificaciones a las leyes laborales que más allá de responder a las necesidades de las organizaciones han generado una pérdida significativa y cuantitativa de las posibilidades de protección social, estabilidad laboral e ingresos económicos para los trabajadores. Suárez, Adriani, Alvariz y Cotignola (2005) se refieren a lo sucedido con la flexibilidad laboral en Argentina, realidad muy cercana a lo que viene sucediendo en Colombia, y expresan que:

...en forma gradual y más lentamente que las modificaciones producidas en el área económica, el Estado fue promoviendo una serie de cambios que eliminaron la estabilidad del empleo y parte de los beneficios que garan- tizaban la protección de los trabajadores: reforma al régimen de contratación temporal, abaratamiento del despido, reducción de aportes patronales a la seguridad social y restricciones a la negociación salarial (Perelman, 2001). De esta manera, se pasó de una legislación laboral de carácter protector a una donde se legitima y refuerza la posición del capital con respecto al trabajo. (Suárez et al., 2005. p. 4).

Isaza citando a Farné y Nupía (2003), agrega que los empresarios para disminuir sus costos optaron por el aumento de la contratación temporal sustituyendo los contratos indefinidos, eliminando las prestaciones extralegales y aumentando los estímulos a la productividad. Cabe enfatizar que esta pérdida en el trabajo ha ido generando un debilitamiento en la calidad de vida de los trabajadores y también en los nexos simbólicos que institucionalmente se habían logrado en el pasado por las mismas organizaciones de trabajo. Lazos que significaban identidad, cohesión y compromiso con los objetivos de la organización.

Bel Adell (1992, p. 183), explica que el término precarización se utiliza para señalar varios elementos que dejan al trabajador en una situación frágil como: a) la duración limitada del contrato temporal; b) los salarios que con frecuencia son inferiores y la ausencia de participación en beneficios no salariales; c) las mayores dificultades en la defensa de los derechos del trabajador y en ocasiones indefensión; d) las peores condiciones de trabajo; y e) la probabilidad de mayores de prácticas contractuales ilegales o abusivas por parte de las empresas.

Citando a Tissera, Suárez et al. (2005, p. 6), afirman que la precarización se manifiesta a través de aspectos económicos tales como los bajos ingresos, las suspensiones, los despidos, la reducción salarial, etc., y en aspectos jurídicos como los tipos de contratación que legalizan formas precarias de inserción laboral, o el incumplimiento de las leyes. Aseguran que el impacto de estas situaciones de inestabilidad, desprotección e inseguridad social y laboral, colocan en una posición desfavorable a la fuerza de trabajo y esto se 
convierte en una intermitencia entre situaciones de ocupación-desocupación-subocupación creciente, según sean las condiciones que dominan en el mercado de trabajo. Estos mismos autores señalan que la labilidad laboral está vinculada con la inseguridad en el empleo y la incertidumbre referida a los ingresos presentes y futuros con los que se puede contar para responder a sus necesidades, asimismo señalan que esto puede conducir a los trabajadores a situaciones de exclusión. Explican la exclusión, citando a Lindenboim (Suárez et al., 2005) al decir que es la incapacidad de las sociedades para integrar a todos sus miembros, especialmente en el sistema económico y al acceso de los beneficios sociales básicos y que se convierte, por tanto, en un impedimento para la integración social y económica. Señala Lindenboim (1997), que quienes logran obtener un puesto de trabajo suelen enfrentarse a un empleo con un plazo limitado y con poca cobertura en materia de seguridad social.

\section{La flexibilidad laboral en Colombia}

Señala Isaza (2003), que la Ley 50 de 1990 constituyó el primer paso en la flexibilización al mercado laboral en Colombia, la cual estuvo orientada a facilitar la creación de nuevos empleos porque hizo menos costosa la contratación y los despidos de los trabajadores. Señala que se propiciaron disposiciones para los contratos temporales y para el despido de quienes llevaban más de diez años en las organizaciones, además se estipuló la forma de los contratos integrales (de más de 10 salarios mínimos legales) y se eliminaron los sobrecostos del regimen de cesantías anterior.

En Colombia se han generado formas de contratación que hacen parte de la flexibilidad laboral, entre ellas están los contratos por misión de las empresas temporales, los trabajos que realizan los asociados a las cooperativas de trabajo asociado, los contratos a término fijo y los contratos de prestación de servicios profesionales. Las personas son afectadas por la inestabilidad laboral, la discontinuidad en la seguridad social y por los limitados ingresos económicos.
Según Pérez y Aragón (1999, p. 27), el contratista sería aquel que ejecute la labor determinada contratada por la empresa y realice también otras actividades diferentes a esta.

Con relación a la contratación temporal, la normatividad colombiana señala en el artículo 71 de la Ley 50 de 1990, que una empresa de servicios temporales es aquella que contrata la prestación de servicios con terceros beneficiarios. Dichas empresas están ubicadas dentro del derecho comercial, el cual refiere el derecho de las empresas. A las organizaciones les conviene este tipo de contratación comercial, porque disminuyen costos administrativos y no tienen que indemnizar a los trabajadores cuando toman la decisión de despedirlo sin justificación (sin justa causa).

En la contratación temporal, la función primordial según la normatividad colombiana es la de cubrir las necesidades de personal que requieran las diferentes compañías, ya sea en los picos de producción (el personal de planta no es suficiente para cubrir la producción), o cuando se deba reemplazar algún empleado por una incapacidad o licencia, ejemplo: la maternidad.

En términos generales las empresas denominadas temporales, que brindan este tipo de contratación, son puentes entre las empresas que requieren personal y los candidatos que se encuentran disponibles en el mercado laboral, es decir, la empresa temporal tiene el rol de ser una intermediaria. Asimismo en el artículo 77 de la Ley 50 de 1990, señala que las empresas usuarias (se denomina usuaria a toda persona natural o jurídica que contrate los servicios de las empresas de servicios temporales) solo podrán contar con personal temporal cuando se trate de las labores ocasionales, accidentales o transitorias; cuando se requiera reemplazar personal que se encuentra en vacaciones, en uso de licencia, en incapacidad por enfermedad o maternidad; para responder ante los incrementos en la producción, el transporte, las ventas de productos o mercancías, en los períodos estacionales de cosechas y en la prestación de servicios, por un término de seis meses, siendo prorrogable hasta por seis meses más. 
Con relación al uso de esta modalidad de contratación comercial que plantea el acceso a trabajadores temporales, en la Tabla 1, se puede apreciar el incremento de utilización de esta forma de contratación en los últimos siete (7) años en Colombia, tanto por actividad económica como por ocupación, se aprecia el incremento desde el año 2003 hasta el año 2009 de la contratación de trabajadores por este medio. Se pasa de contratar 401.797 trabajadores en el año 2003 a 489.415 en el año 2004; 697.363 en el 2005 a 621.984 en el 2006; 751.920 en el 2007 a 834.057 en el 2008 y 866.158 en el 2009.

Tabla 1.

Demanda de mano de obra (colocaciones) a través de las Empresas de Servicios Temporales según actividad económica de la empresa solicitante - Serie Anual, 2003-2009.

\begin{tabular}{|c|c|c|c|c|c|c|c|c|}
\hline Actividad económica & 2003 & 2004 & 2005 & 2006 & 2007 & 2008 & 2009 & TOTAL \\
\hline $\begin{array}{l}\text { Agricultura. Ganadería. } \\
\text { Servicultura. Caza y } \\
\text { Pesca }\end{array}$ & 19.021 & 18.782 & 73.903 & 34.674 & 59.190 & 65.852 & 72.056 & 343.478 \\
\hline $\begin{array}{l}\text { Explotación de Minas y } \\
\text { Canteras }\end{array}$ & 12.143 & 30.612 & 44.386 & 23.366 & 19.637 & 17.770 & 46.672 & 194.586 \\
\hline Industria Manufacturera & 129.733 & 138.366 & 162.672 & 163.288 & 176.045 & 165.382 & 151.884 & 1.087.370 \\
\hline $\begin{array}{l}\text { Electricidad, Agua, Gas } \\
\text { y Vapor }\end{array}$ & 13.046 & 17.070 & 46.414 & 19.227 & 21.797 & 63.898 & 49.892 & 231.344 \\
\hline Construcción & 12.861 & 26.107 & 48.190 & 21.987 & 35.100 & 42.513 & 74.005 & 260.763 \\
\hline $\begin{array}{l}\text { Comercio al por mayor } \\
\text { y al por menor }\end{array}$ & 81.006 & 82.277 & 71.788 & 101.116 & 116.265 & 116.748 & 109.314 & 678.514 \\
\hline $\begin{array}{l}\text { Transportes y } \\
\text { Comunicaciones }\end{array}$ & 20.765 & 22.512 & 34.299 & 30.457 & 41.247 & 50.438 & 62.483 & 262.201 \\
\hline $\begin{array}{l}\text { Establecimientos } \\
\text { Financ. Seg. Bienes } \\
\text { Inmuebles y Ss. }\end{array}$ & 46.240 & 60.618 & 84.625 & 65.298 & 63.851 & 81.032 & 104.358 & 506.022 \\
\hline Servicios Comerciales & 45.858 & 69.110 & 88.342 & 118.298 & 139.577 & 150.754 & 123.329 & 735.268 \\
\hline $\begin{array}{l}\text { Actividades no bien } \\
\text { especificadas }\end{array}$ & 21.124 & 23.961 & 42.744 & 44.273 & 79.211 & 79.670 & 72.165 & 363.148 \\
\hline TOTAL & 401.797 & 489.415 & 697.363 & 621.984 & 751.920 & 834.057 & 866.158 & 4.662 .694 \\
\hline
\end{tabular}

Nota: Minprotección Social - Direcciones Territoriales de Trabajo - Informes Trimestrales EST. Las colocaciones de mano de obra están constituidas por el número de contratos que efectivamente fueron realizados a través de las Empresas de Servicios Temporales (EST). Las empresas usuarias se clasifican según la actividad económica que desempeñan. Última actualización: octubre 5 de 2010.

Entre las actividades económicas, desde el año 2003 al 2009, los trabajadores que más se contratan bajo esta modalidad de relación contractual están: industria manufacturera $11^{`} 087.370$ de trabajadores; servicios comerciales: 735.268; comercio al por mayor y al detal: 678.514; y establecimientos financieros, seguros, bienes inmuebles: 506.022 .

Según la Tabla 2, las ocupaciones más requeridas para laborar en empresas temporales son: oficinistas y auxiliares: 1'351.607; ayudantes de procesamiento y fabricación: 917.900; ocupaciones elementales en ventas y servicios: 828.011 ; ocupaciones administrativas: 628.448; técnicos en ventas y servicios: 507.150 ; operadores de máquinas de procesamiento y fabricación y ensambladores: 448.022; ocupaciones intermedias en ventas y servicios: 396.879 .

Igualmente se aprecia el incremento de los empleados por este tipo de contratación, y cómo las empresas temporales intermedian personal poco calificado, semicalificado y calificado, es decir, de todo tipo de personal. Cabe señalar que esta es una realidad que es para todo tipo de trabajadores y no solo se trata de personal poco calificado. 
Tabla 2.

Oferta de mano de obra a través de las empresas de servicios temporales según la clasificación nacional de ocupaciones.

\begin{tabular}{|c|c|c|c|c|}
\hline Clasificación nacional de ocupaciones & 2007 & 2008 & 2009 & TOTAL \\
\hline Alta dirección & 2.712 & 4.679 & 5.045 & 23.259 \\
\hline Gerencia Media & 4.859 & 11.044 & 5.593 & 53.630 \\
\hline Profesionales en administración y finanzas & 33.944 & 67.332 & 43.978 & 264.891 \\
\hline Ocupaciones administrativas & 113.964 & 147.926 & 130.167 & 628.448 \\
\hline Oficinistas y auxiliares & 204.503 & 271.364 & 237.407 & $1 ` 351.607$ \\
\hline Profesionales en ciencias naturales y aplicadas & 4.855 & 9.778 & 6.738 & 42.973 \\
\hline Técnicas de las ciencias naturales y aplicadas & 2.622 & 2.840 & 3.111 & 18.491 \\
\hline Profesionales en salud & 36.835 & 10.767 & 39.111 & 262.678 \\
\hline Técnicos en salud & 26.899 & 5.994 & 27.493 & 196.476 \\
\hline Auxiliares en servicios de la salud & 36.761 & 29.628 & 38.984 & 240.337 \\
\hline $\begin{array}{l}\text { Profesionales en ciencias sociales, educación, servicios } \\
\text { gubernamentales y religión }\end{array}$ & 2.578 & 4.158 & 4.750 & 21.377 \\
\hline Nivel medio en servicio social, educación y religión & 1.642 & 2.253 & 2.656 & 13.955 \\
\hline Ocupaciones profesionales en arte y cultura & 863 & 1.158 & 1.680 & 7.932 \\
\hline $\begin{array}{l}\text { Técnicos y especializadas en arte, cultura, esparcimiento } \\
\text { y deporte }\end{array}$ & 2.822 & 3.823 & 4.741 & 21.715 \\
\hline Ocupaciones profesionales en ventas y servicios & 33.503 & 56.976 & 54.008 & 312.188 \\
\hline Técnicos en ventas y servicios & 76.834 & 96.920 & 105.769 & 507.150 \\
\hline Ocupaciones intermedias en ventas y servicios & 57.536 & 51.553 & 54.278 & 396.879 \\
\hline Ocupaciones elementales en ventas y servicios & 115.449 & 152.055 & 157.108 & 828.011 \\
\hline Técnicos de la actividad primaria y extractiva & 21.432 & 15.869 & 20.897 & 85.981 \\
\hline $\begin{array}{l}\text { Ocupaciones intermedias en la actividad primaria y } \\
\text { extractiva }\end{array}$ & 19.291 & 11.730 & 13.577 & 85.702 \\
\hline Obreros de la actividad primaria y extractiva & 34.879 & 35.078 & 37.744 & 229.571 \\
\hline $\begin{array}{l}\text { Contratistas y supervisores de oficios y de operadores de } \\
\text { equipo y transporte }\end{array}$ & 10.475 & 12.038 & 19.594 & 89.381 \\
\hline Oficios y operadores de equipos y transporte & 58.473 & 58.605 & 54.907 & 384.405 \\
\hline Obreros y ayudantes de la construcción y de otros oficios & 36.903 & 39.027 & 40.264 & 185.504 \\
\hline $\begin{array}{l}\text { Supervisores y ocupaciones técnicas en procesamiento } \\
\text { y fabricación (Superv. y Ocup. Técn. en Proc. Fabric y } \\
\text { Sumin Ss Públ.) }\end{array}$ & 11.809 & 12.831 & 11.239 & 80.685 \\
\hline $\begin{array}{l}\text { Operadores de máquinas de procesamiento y fabricación } \\
\text { y ensambladores }\end{array}$ & 59.366 & 81.610 & 64.308 & 448.022 \\
\hline Ayudantes de procesamiento y fabricación & 108.866 & 128.961 & 154.777 & 917.900 \\
\hline TOTAL & $1 ` 120.675$ & $1 ` 325.997$ & $1 ` 348.187$ & $7 ` 707.411$ \\
\hline
\end{tabular}

Nota: Tomado y adaptado de Minprotección Social - Direcciones Territoriales de Trabajo - Informes Trimestrales EST. Última actualización: octubre 5 de 2010.

En la Tabla 3, Cantidad de Empresas Temporales en Colombia por Departamento, se puede apreciar la cantidad de empresas temporales que existen en el país por departamento, en el año 2013. Los que más tienen son Cundinamarca con
261 (incluye la capital de Colombia, Bogotá); Antioquia (incluye su capital Medellín) con 82; Atlántico con 87 y Valle del Cauca con 58. En Colombia en total hay 595 empresas temporales y $221 \mathrm{Su}$ cursales. 
Tabla 3

Cantidad de Empresas Temporales Colombianas por Departamento.

\begin{tabular}{|c|c|c|}
\hline Departamento & $\begin{array}{c}\text { Sedes } \\
\text { principales }\end{array}$ & Sucursales \\
\hline Amazonas & 1 & \\
\hline Antioquia & 42 & 40 \\
\hline Arauca & 1 & \\
\hline Atlántico & 83 & 4 \\
\hline Bolívar & 29 & 4 \\
\hline Boyacá & 2 & \\
\hline Caldas & 5 & 16 \\
\hline Caquetá & 1 & \\
\hline Casanare & 4 & 2 \\
\hline Cauca & 3 & \\
\hline Cesar & 15 & 7 \\
\hline Choco & & 2 \\
\hline Córdoba & 12 & 6 \\
\hline Cundinamarca & 261 & 24 \\
\hline Guajira & 5 & 1 \\
\hline Huila & 7 & 7 \\
\hline Magdalena & 7 & 10 \\
\hline Meta & 7 & 7 \\
\hline Nariño & 5 & 1 \\
\hline Norte de Santander & 10 & 3 \\
\hline Quindío & 8 & 6 \\
\hline Risaralda & 10 & 28 \\
\hline San Andres Isla & 2 & 1 \\
\hline Santander & 20 & 13 \\
\hline Sucre & 5 & 1 \\
\hline Tolima & 8 & 22 \\
\hline Valle del Cauca & 42 & 16 \\
\hline Total & 595 & 221 \\
\hline
\end{tabular}

Nota: Construcción propia de los datos obtenidos del Registro Nacional de empresas de servicios temporales - junio 2013. Ministerio del Trabajo.

La otra forma de flexibilidad laboral expresada en la forma de contratación y que está siendo muy utilizada en Colombia, son las cooperativas de trabajo asociado, que se crearon con el objetivo de asociar a un grupo de trabajadores en pro del mejoramiento de su calidad de vida mediante el ofrecimiento de su fuerza laboral, objetivo que se desdibujo porque buena parte de las cooperativas de trabajo asociado operó en función del logro de lucro a través de la asociación laboral.

En la Figura 1, se puede apreciar cómo hasta mediados de la década de los ochenta el número de cooperativas de trabajo asociado creadas por año no superaba el promedio de dos (2); posteriormente, hasta mediados de la década de los noventa, el promedio de cooperativas creadas por año subió a 20 y para la segunda mitad de la década de los noventa dicho promedio llegó a 125 cooperativas creadas por año. Pero es luego del año 2000 cuando la proliferación de las cooperativas de trabajo asociado en Colombia se da con mayor fuerza, particularmente durante los años 2002, 2003 y 2004 (Observatorio cooperativo, 2009).

Con la Ley 79 de 1988, el Gobierno definió la naturaleza de las cooperativas y precooperativas de trabajo asociado, en el artículo 59 dice:

Las cooperativas de trabajo asociado en que los aportantes de capital son al mismo tiempo los trabajadores y gestores de la empresa, el régimen de trabajo, de previsión, seguridad social y compensación, será establecido en los estatutos y reglamentos en razón a que se originan en el acuerdo cooperativo y, por consiguiente, no estará sujeto a la legislación laboral aplicable a los trabajadores dependientes.

Asimismo en el artículo 3 del Decreto 2879 de 2004, definió que constituye una práctica prohibida y no autorizada la prestación de servicios a terceros cuando una persona natural o jurídica, las cooperativas y precooperativas de trabajo asociado o una Empresa Asociativa de Trabajo prestan servicios en los eventos específicamente previstos por el artículo 77 de la Ley 50 de 1990 para las Empresas de Servicios Temporales.

El gobierno puso en vigencia la Ley 1233 de 2008, que reglamenta la actividad de las llamadas cooperativas y precooperativas de trabajo asociado 
Figura 1. Cooperativas de trabajo asociado creadas desde 1966 hasta 2008.

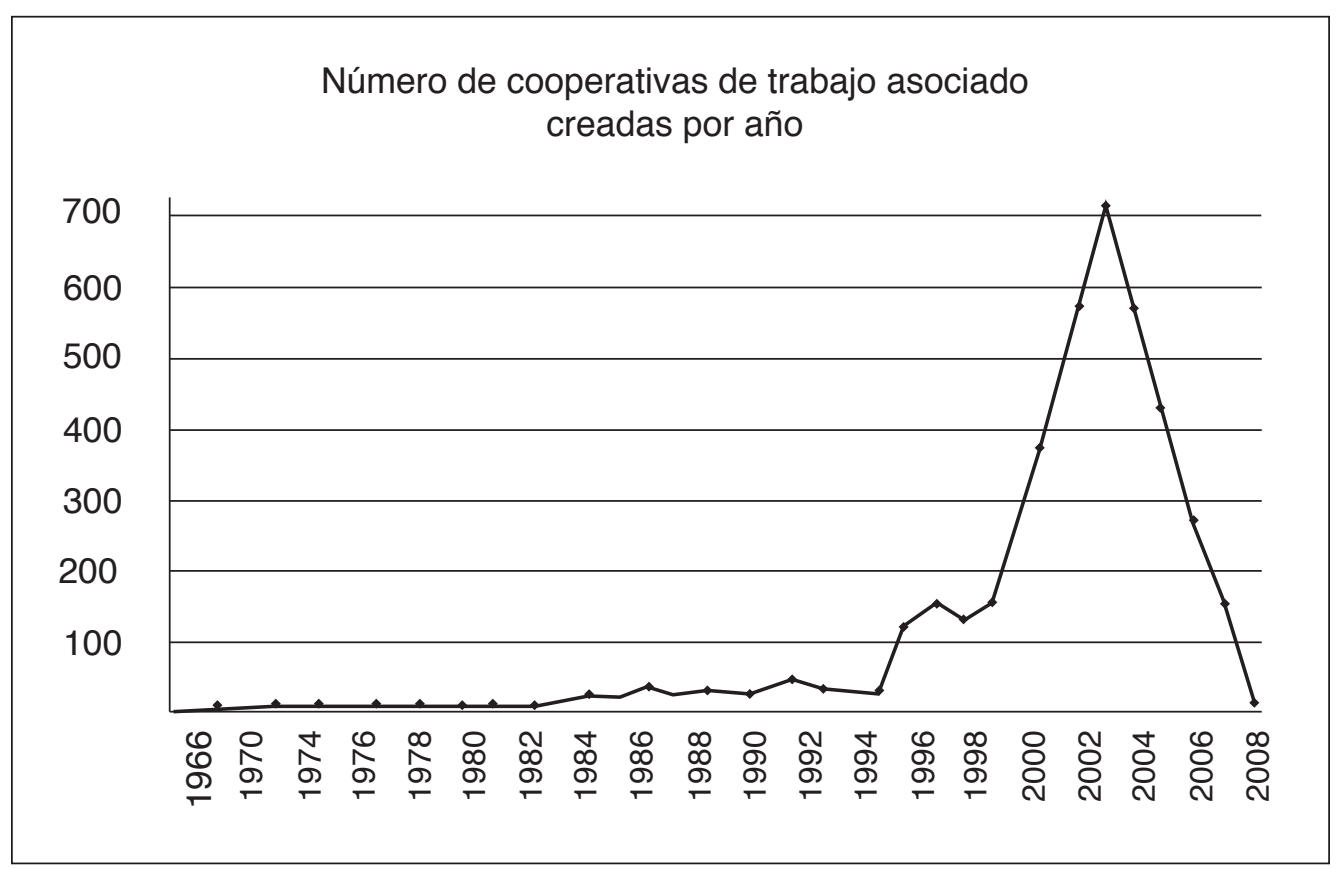

Nota: Observatorio cooperativo. Cooperativas de trabajo asociado. Agosto 2009.

con la pretensión de poner fin a la intermediación laboral en el país. Con la ley busca que las cooperativas garanticen al asociado un ingreso mensual básico equivalente al menos al salario mínimo legal vigente y el pago de los aportes a la seguridad social de los trabajadores vinculados, así como de las contribuciones parafiscales al Sistema Nacional de Aprendizaje-SENA, Instituto de Bienestar Familiar-ICBF y Cajas de Compensación.

Se estima que en Colombia funcionan legalmente tres mil quinientas cooperativas de trabajo asociado y se calcula que entre siete a ocho mil lo hacen informalmente. Entre 500 a 600 mil trabajadores asociados se beneficiarán con la nueva Ley de la República (Caracol Radio, julio 2008).

En la Tabla 4, se aprecia el gran número de cooperativas y precooperativas de trabajo asociado, 5.664; de las cuales 4.089 estaban en regla, 1.315 estaban en trámite para su funcionamiento, 76 fueron objetadas y 13 archivadas, para el año 2010.
El Ministerio de Protección Social (actualmente Ministerio del Trabajo, separado mediante la ley 1444 de 2011, del Ministerio de Salud y Protección Social), y la Superintendencia de la Economía Solidaria, en la Circular 0067 del 2005, realizaron una distinción entre las Cooperativas de Trabajo Asociado (CTA) y las Empresas de Servicios Temporales (EST), porque bajo la figura de Trabajo Asociado se venían constituyendo una gran cantidad de cooperativas y precooperativas para desarrollar inapropiadamente su objeto social, ofreciendo actividades propias de las Empresas de Servicios Temporales o para operar como Agrupadoras en Salud. Esto era contrario a lo estipulado en la Ley 79 de 1988 y Decreto 468 de 1990; Ley 50 de 1990, Decretos 024 de 1998, 503 de 1998, 1703 y 2400 de 2002.

Esta circular tenía por objetivo hacer un llamado a la responsabilidad social, que se espera, deba prevalecer en los agentes del mercado laboral como empleadores, empresarios, asesores de empresas y trabajadores. Ese llamado de atención buscaba hacer un adecuado uso de las Cooperativas de 
Tabla 4.

Cooperativas y precooperativas de trabajo asociado.

\begin{tabular}{|c|c|c|c|c|c|}
\hline Departamento & CTA & Resuelta & En trámite & Objetada & Archivada \\
\hline Amazonas & 6 & 6 & 0 & 0 & 0 \\
\hline Antioquia & 488 & 106 & 176 & 35 & 0 \\
\hline Arauca & 21 & 21 & 0 & 0 & 0 \\
\hline Atlántico & 374 & 276 & 94 & 4 & 0 \\
\hline Bolívar & 98 & 83 & 14 & 1 & 0 \\
\hline Boyacá & 102 & 75 & 27 & 0 & 0 \\
\hline Caldas & 111 & 107 & 4 & 0 & 0 \\
\hline Caquetá & 16 & 13 & 3 & 0 & 0 \\
\hline Casanare & 30 & 30 & 0 & 0 & 0 \\
\hline Cauca & 197 & 165 & 32 & 0 & 0 \\
\hline Cesar & 146 & 128 & 1 & 17 & 0 \\
\hline Chocó & 18 & 18 & 0 & 0 & 0 \\
\hline Córdoba & 79 & 53 & 26 & 0 & 0 \\
\hline Cundinamarca & 1.345 & 1.063 & 275 & 0 & 7 \\
\hline Guainía & 1 & 0 & 1 & 0 & 0 \\
\hline Guaviare & 7 & 7 & 0 & 0 & 0 \\
\hline Guajira & 41 & 41 & 0 & 0 & 0 \\
\hline Huila & 195 & 181 & 14 & 0 & 0 \\
\hline Magdalena & 115 & 100 & 15 & 0 & 0 \\
\hline Meta & 194 & 166 & 22 & 0 & 6 \\
\hline Nariño & 180 & 173 & 7 & 0 & 0 \\
\hline Norte de Santander & 158 & 143 & 15 & 0 & 0 \\
\hline Putumayo & 32 & 21 & 11 & 0 & 0 \\
\hline Quindío & 71 & 67 & 4 & 0 & 0 \\
\hline Risaralda & 116 & 98 & 18 & 0 & 0 \\
\hline San Andrés Isla & 3 & 3 & 0 & 0 & 0 \\
\hline Santander & 616 & 470 & 146 & 0 & 0 \\
\hline Sucre & 48 & 35 & 11 & 2 & 0 \\
\hline Tolima & 109 & 98 & 11 & 0 & 0 \\
\hline Valle del cauca & 746 & 341 & 388 & 17 & 0 \\
\hline Vaupés & 1 & 1 & 0 & 0 & 0 \\
\hline Total & 5.664 & 4.089 & 1.315 & 76 & 13 \\
\hline
\end{tabular}

Nota: Construcción propia a partir de la información obtenida del Ministerio de Protección Social. Registro de cooperativas y precooperativas de trabajo asociado de 2007 y 2008 . (Estado de radicación: Resuelta, En trámite, Objetada y Archivada). Introducción. Republica de Colombia. $3^{\text {a }}$ edición.

Trabajo Asociado, de acuerdo con su naturaleza y que se evitara convertirlas en fuentes de evasión de impuestos, parafiscales o empobrecimiento de la fuerza de trabajo, actividades, que señalaron, generan responsabilidades administrativas y penales por violación a la ley.

Lo lamentable de esto, es que se dictan leyes pero en la realidad la precarización laboral es un tema real, no solo porque muchos ven más limita- das sus posibilidades de estabilidad laboral y desarrollo de su potencial, sino porque su capacidad de ser integrantes de una comunidad de trabajo es supremamente frágil y están constantemente en una situación de incertidumbre, y en el lugar de "desecho", pues pueden ser despedidos sin las más mínimas consideraciones.

A finales del año 2010, al acercarse las fechas de concertación entre el gobierno, las centrales 
obreras y los representantes de las empresas, para acordar el salario mínimo legal para el año 2011, el Vicepresidente de la República Colombiana, Angelino Garzón (quien ha hecho parte del sindicalismo en el pasado), señaló la necesidad de eliminar las cooperativas de trabajo asociado por la pérdida de seguridad social que están produciendo en el país.

Bogotá. "Pido a la Comisión que revise el funcionamiento de las denominadas Cooperativas de Trabajo Asociado. Los principales violadores de los derechos laborales son estas cooperativas. Es injusto que los trabajadores que prestan servicios a través de estas no estén protegidos por las leyes laborales, sino por el régimen comercial", dijo. De acuerdo con el Vicepresidente, estas cooperativas de trabajo asociado "solo sirven para facilitar empleos precarios", llevando así a que en Colombia "haya un retroceso de más de doscientos años". "Las cooperativas de trabajo asociado se han convertido en el mejor símbolo de la sobre-explotación de los trabajadores y de la violación de los derechos laborales en Colombia", dijo el Vicepresidente (periódico La Republica, noviembre 4 de 2010).

Esta confirmación que el Vicepresidente le dio a esta realidad colombiana, es fundamental para realizar cambios y ajustes importantes para diseñar políticas públicas que también protejan socialmente a los trabajadores, además de establecer políticas que ayuden a la economía del país.

Otra forma de flexibilización relacionada con los tipos de contratos laborales, es la del contrato a término fijo. En esta se muestra cómo el patrono puede optar por no renovar el contrato aun cuando subsistan las causas que originaron el contrato inicialmente, es decir, así necesite personal para desempeñar dicho cargo. Solo en algunos casos especiales y excepcionales, y en los que se pruebe que la no renovación del contrato obedece a una discriminación por su condición física, de salud o laboral, puede alegarse la ilegalidad de la decisión de no renovar el contrato. Es importante señalar que se aprecia una tendencia cada vez mayor a la utilización de contratos fijos sobre los indefinidos.
En caída contratos de trabajadores a término indefinido. Al mismo tiempo, y como la otra cara de la moneda, los empleadores no se sienten animados a vincular trabajadores de manera indefinida, bien sea porque con otras modalidades les resultan más baratos -y mejoran su competitividad y su estado de pérdidas y ganancias- o porque las expectativas de un desempeño empresarial sostenido y creciente no son lo suficientemente altas (Red de Gestores Sociales. Periódico Portafolio. Agosto 4 de 2008).

Sobre la modalidad de contrato que se viene utilizando para servicios profesionales, denominada contrato de prestación de servicios, el cual está regulado por el código civil, pero no por el laboral. Se caracteriza porque el contratista presta el servicio contratado con plena autonomía financiera, administrativa y técnica, a cambio de una suma de dinero pagadera según pacten las partes, así como el valor, la forma de pago, las obligaciones de las partes, el término de duración, las causales de terminación, etc. El que contrata el servicio se denomina contratante y el que lo presta contratista. Pueden realizarse contratos de prestación de servicios con personas naturales, como en el caso del abogado, contador, etc.

Afirma Rodríguez (2007), que La ley 789 de 2002, es una parte primordial de la política de flexibilización laboral y que está relacionada con las tendencias generadas por la globalización y la firme internacionalización de la economía, la cual implantó cambios importantes en las relaciones laborales, al hacer una reducción de las indemnizaciones por despido injusto, reducir el pago de horas extras y disminuir los recargos por festivos y dominicales.

Ante la problemática del desempleo y sus consecuencias sociales adversas, y la urgencia de las organizaciones de ser más competitivas, es que se sancionó la Ley 789 de 2002, conocida como la Ley de Empleabilidad y Protección Social, que empezó a regir en el año 2003 y que prometía garantizar una mayor equidad entre los colombianos, y con la cual se esperaba generar alrededor de 600.000 nuevos empleos en los siguientes cuatro años. 
Esta reforma laboral finalmente introdujo cambios en la normatividad laboral en beneficio de las empresas, pero con miras también a incrementar el empleo. La reforma modificó la jornada de trabajo para que esta fuera más flexible a las necesidades del empleador; asimismo lo que se considera trabajo ordinario y nocturno, pues se extendió el horario diurno hasta las 10 p. m., para que el empleador no pagará recargo nocturno de las 6:00 a las 10 p. m.; se modificó el recargo por el trabajo de domingos y festivos; además se disminuyeron los costos para las organizaciones con relación a las indemnizaciones por despido sin justa causa. Cabe señalar que pasados cuatro años de haber sido generada esta Ley, se realizaron estudios que llevaron a decir que dicha reforma no incrementó el empleo y sí precarizó el trabajo.

El estudio más reciente sobre la reforma laboral de 2002, fue publicado en abril de 2007, por el Centro de Investigaciones para el Desarrollo de la Facultad de Ciencias Económicas de la Universidad Nacional. Su diagnóstico señala que no existen pruebas convincentes de que los movimientos de la tasa de desempleo hacia la baja hayan sido ocasionados por una demanda laboral, y que tampoco es cierto que se aprecie una tendencia sostenida en el aumento del empleo informal. En otras palabras, no hay indicadores que demuestren que la reforma produjo cambios en el mercado (periódico El Tiempo, febrero 22 de 2008).

En contraste, dice Rodríguez (2007) haciendo referencia a los tratados de libre comercio -TLC-, que viene buscando Colombia establecer con otros países, es claro que el aspecto laboral y la observancia de los estándares laborales internacionales, tienen cada vez mayor peso dentro de las negociaciones, como ya se evidenció en el proceso para lograr un TLC con Estados Unidos. Señala que de estos acuerdos e instrumentos creados en el ámbito internacional es que se ha planteado una estandarización de la normatividad laboral con base en las disposiciones de la OIT, de la cual Colombia tendrá que acogerse.

En los últimos años en Colombia se trabajó por lograr firmar un tratado de libre comercio ${ }^{1}$ con
Estados Unidos, y una de las exigencias que hizo el presidente de este país, fue la de revisar los derechos laborales, el Presidente Santos expresó su compromiso para poder firmar a futuro dicho TLC.

El presidente estadounidense, Barack Obama, recibe este jueves a su homólogo colombiano Juan Manuel Santos en la Casa Blanca para darle un nuevo impulso a las relaciones bilaterales y despejar el camino al Tratado de Libre Comercio (TLC), pendiente entre ambos países..."En su segundo encuentro desde que Santos asumió el poder, ambos presidentes anunciarán un plan para reforzar los derechos laborales en Colombia, uno de los países donde se cometen más asesinatos de sindicalistas en el mundo" (Periódico El Espectador, abril 7 de 2011).

La flexibilización laboral en el contexto colombiano ha traído consecuencias contraproducentes para los trabajadores, e incluso para el ambiente laboral de las organizaciones, pues la inequidad es parte del ambiente de trabajo, ejemplo de ello es que no todos quienes trabajan en una organización, sean trabajadores directos o externalizados, acceden a la capacitación, auxilios, bonificaciones, estabilidad u otros servicios, así compartan los mismos lugares de labor.

Afirman Farné y Vergara (2007), que en Colombia se han incrementado las formas de regulación laboral que liberan a los empleadores de toda responsabilidad con sus colaboradores y desplazan por completo el costo de la responsabilidad social sobre el trabajador o sobre unos terceros intermediarios. Además enfatizan, que el crecimiento económico del país en los últimos años no ha sido capaz de reducir la informalidad urbana por debajo del $60 \%$, y tampoco ha logrado un impacto potente sobre la calidad del trabajo para los colombianos. Igualmente expresa Rodríguez (2007), que se viene presentando una relajación en las relaciones de trabajo a razón del aumento en la subcontratación, la temporalidad y el trabajo relacionado a las cooperativas y a los intermediarios u outsourcing, porque por estas vías las empresas han entregado sus obligaciones laborales a terceros y en otros casos, a los mismos trabajadores. 
Agrega Rodríguez (2007) que para muchas empresas, la observancia de los estándares laborales se convierte cada vez más en un factor de competitividad decisivo y lleva a una revisión de muchas de las medidas de flexibilización implementadas, para garantizar una mejor inserción en los mercados internacionales. Es decir, que todo no esta dicho y concluido en este escenario de relaciones laborales, las consecuencias de la normatividad y de los controles establecidos y aplicados, posibilitarán una revisión y ajuste a partir de los análisis de las realidades que permitan argumentos agudos que favorezcan nuevas propuestas que evolucionen a un escenario al que le urge considerar además de las necesidades y urgencias de las organizaciones, las de quienes las constituyen, las crean y las mantienen, ya como trabajadores o como quienes también hacen uso de ellas por sus servicios o productos, porque si ellos no tienen acceso al trabajo o empleo de calidad, ¿cómo será posible que "consuman" sus productos o servicios?

\section{Discusión}

Es importante señalar que los empleadores contratan y desvinculan al personal bajo un criterio básicamente económico, en la lógica de lograr una mayor ganancia, esto en sí mismo no es el problema. Lo problemático es la lógica de un discurso que privilegia el beneficio de quien tiene los medios sobre otros que les urge un empleo para la supervivencia, su desarrollo e incluso su goce presente y futuro logrado a través del trabajo y del salario percibido. Es una lógica perversa que precariza el trabajo y disimula su saber sobre el costo que tiene para la relación social, el bienestar y la llamada calidad de vida de las personas que integran la sociedad.

En muchos casos existen las leyes laborales que pretenden responder a la dinámica mundial de competitividad, pero el desconocimiento y la aplicación irregular de las mismas por los empleadores y la falta de control, de incluso su cumplimiento por parte del Estado han favorecido la pérdida de los derechos de los trabajadores, muestra de ello ha sido lo que pasó por aproxi- madamente una década, con las cooperativas de trabajo asociado.

Si las razones por las cuales se opta por la flexibilización del trabajo fueran solo de la importancia de la competitividad y del incremento del empleo, esta reflexión sería poco importante, pero si de lo que se trata es además de estudiar cómo esta realidad, también social y no solo económica, es producida por el discurso del capitalismo, y tiene efectos en los sentidos y significados del trabajo para los trabajadores, las empresas y el Estado, y que tiene serias repercusiones en la vida de las personas en su contexto laboral y social, se hace además de necesario, urgente.

Si bien hay un entorno que presiona sobre los trabajadores y las organizaciones, también es cierto que hay que estudiar cómo estos cambios producen sufrimiento, establecen relaciones poco equitativas y fomentan el exceso del poder del más fuerte. El trabajo es una parte importante de la vida de las personas y reducirla solo a la expresión de la urgencia por la sobrevivencia, es un retroceso en el avance de la racionalidad humana y de su proceso mismo de humanización.

\section{Referencias}

Bel, C. (1992). Flexibilización del mercado de trabajo y precarización del empleo: la contratación temporal; aspectos teóricos, legales e históricos. Investigaciones geográficas, 10, 181-196.

Caracol Radio. (2008). Por Ley de la República prohíben en Colombia la intermediación laboral. Recuperado de http://www.caracol. com.co/nota. aspx?id=638080

Dane. (2010). Colombia. Proyecciones de Población Municipales por área 2005-2020. Recuperado de http://www.dane.gov.co/daneweb_V09/index.php?option=com_content\& view $=$ article\&id $=75 \&$ Itemid $=72$

El Espectador, periódico. (2011). Recuperado de http://www.elespectador.com/economia/ articulo-261482-obama-recibe-santos-desbloquear-el-tlc-entre-eeuu-y-colombia 
Farné, S., Vergara, C. A. (2007). Calidad del empleo: ¿qué tan satisfechos están los colombianos con su trabajo? Cuyuntura social. 36, 51-69.

Fernández, L. (2006). Flexibilización y precarización laboral. Fundación Escuela de Gerencia Social. Caracas: Ministerio de Poder Popular para la Planificación y Desarrollo. Recuperado de http://fegs.msinfo.info/fegs/archivos/ pdf/FPL.PDF

Gerencie.com. (2008). Cooperativas de Trabajo Asociado; un golpe bajo al trabajador Colombiano. Recuperado de http://www.gerencie. com/cooperativas-de-trabajo-asociado-ungolpe-bajo-al-trabajador-colombiano.html

Isaza, J. G. (2003). Flexibilización laboral: un análisis de sus efectos sociales para el caso Colombiano. Equidad y Desarrollo, 1, 9-39.

Mària, S., Joseph, F. (2000). El "Consenso de Washington" ¿Paradigma Económico del Capitalismo Triunfante? Revista de fomento social. 217, 29-45.

Ministerio del Trabajo. República de Colombia. Registro de Empresas de Servicios Temporales (2013). Recuperado de http://www. mintrabajo.gov.co/buscar.html?searchword= REGISTRO+DE+EMPRESAS\&ordering=\&search phrase $=$ all

La Republica, periódico. (2010). Angelino Garzón pidió que se acaben las cooperativas de trabajo asociado. Recuperado de http:// www.larepublica.com.co/archivos/ECONOMIA/2010-11-04/angelino-garzon-pidio-quese-acaben-las-cooperativas-de-trabajo-asociado_114458.php\#

Lindenboim, J. (1997). Desocupación, precariedad y flexibilidad laboral: un debate abierto. Interamericana de Planificación, 113, 29-47.
Nullvalue. (2008). En caída contratos de trabajadores a término indefinido. Publicación Portafolio.com.co. Sección información general. Recuperado de http://www.rgs.gov.co/noticias.shtml?x=24153

Rodríguez, C. (2007). ¿Competir con el empleo precario?, Economía Colombiana, 320,50-63,

Sierra, H. (2008). Tambalea la Reforma Laboral. Periódico El Tiempo. Recuperado de: http:// www.elespectador.com/impreso/cuadernilloa/judicial/articuloimpreso-tambalea-reforma-laboral-corte.

Suárez, M. J.; Adriani, H. L.; Alvariz, A; Cotignola, M. (2005). En Principales características de la precarización laboral en el gran la plata. Período 2003-2004, Séptimo congreso nacional de estudios del trabajo. Recuperado de http://www.aset.org.ar/congresos/7/09006. pdf

Observatorio cooperativo Coofecop. (2009). Cooperativas de trabajo asociado. Recuperado de http://www.portalcooperativo.coop/observatorio/docs/doc11.pdf

OIT. (2002). Conferencia Internacional del Trabajo 90a reunión 2002. Recuperado de http:// www.ilo.org/public/spanish/standards/relm/ ilc/ilc90/pdf/rep-vi.pdf

Paz, R.; Piedrahita, M. (2006). Flexibilización laboral. En el sector farmacéutico de Colombia. Universidad Libre, Seccional Cali. Cali: Ed. Universidad Libre.

Pérez, G., Aragón de Pérez, V. (1999). El servicio temporal en Colombia. En: Flexibilización laboral y Outsourcing. Santafé de Bogotá: Ed. Biblioteca Jurídica Diké.

Presidencia de la República. (2006). Recuperado de http://www.presidencia.gov.co/tlc/documentos/abc1.htm 